\title{
PORFIRIA AGUDA Y EMBARAZO
}

\section{REVISION DEL TEMA Y PRESENTACION DE UN CASO}

\section{Doctor Jaime Ramírez Sánchez (*)}

A pesar de haberse escrito mucho sobre porfiria intermitente aguda, poco se ha mencionado esta enfermedad en asociación con єmbarazo. Una revisión de la literatura revela solamente 14 casos de embarazos o puerperios complicados con porfiria intermitente aguda. De éstos, una madre murió en una recaída 8 días después del parto; la orina del niño al nacer, en este caso, era color vino Oporto, positiva para uroporfirina y contenía una cantidad excesiva de coproporfirina. Sin embargo, aclaró rápidamente y poco ciespués fue normal. Aunque el niño pueda haber formado estos pigmentos rojos en exceso, la posibilidad de que ellos hayan atravesado la placenta no puede ser despreciada.

De enero 1 o a diciembre 31 de 1955 , se encontraron 4 casos de porfiria intermitente aguda complicada con embarazo en el servicio obstétrico del King's County Hospital de Brooklyn, New York. Durante este período de tiempo hubo un total de 5.300 nacimientos, para una incidencia de 1:1328. Sin embargo, en 1956, sobre 8.300 nacimientos no se encontró ningún caso, lo cual hace pensar que la incidencia indicada arriba sea mucho mayor que la incidencia real.

Dada la rareza de la entidad consideramos conveniente hacer una rápida revisión del tema de las porfirias.

"Se conoce con el nombre de porfirias a un grupo de enfermedades caracterizadas por alteración del metabolismo de las porfirinas, con aumento en la eliminación de uroporfirinas o de

(*) Asistente de Cátedra de Clínica Obstétrica de la Facultad de Medicina de la Universidad Nacional. 
sus precursores en la orina y las heces. Aunque poco se sabe de su etiología y patogenia, los estudios más recientes tienden a demostrar su carácter hereditario y así actualmente se clasifican como "errores congénitos del metabolismo", según la noción expuesta inicialmente por Garrod.

Las porfirinas son compuestos heterocíclicos formados por la unión de cuatro anillos pirrólicos, mediante cuatro enlaces de meteno y constituyen el núcleo prostético de los pigmentos respiratorios: hemoglobina, mioglobina, citocromo, catalasa y peroxidasa. Igualmente se hallan en la molécula de clorofila, la cual $\epsilon S$ un compuesto de porfirina y magnesio.

La estructura heterocíclica formada por la unión de cuatro anillos pirrórillos mediante cuatro puentes de meteno, se conoce con el nombre de porfina. En esta estructura los anillos carbóricos presentan ocho carbonos unidos a otros tantos átomos de hidrógeno, los cuales pueden ser reemplazados por radicales diferentes, obteniéndose así los distintos tipos de porfirinas. Al reemplazar los hidrógenos por cuatro metilos y cuatro etilos, se obtiene la etioporfirina, compuesto que no se encuentra en la naturaleza, siendo exclusivamente productos de laboratorio.

Según la posición relativa de los diferentes radicales, son posibles cuatro isómeros, que se identifican con los numerales I a IV. Es de anotarse que en la naturaleza solo se encuentran los derivados de los porfirinas I y III, siendo el tipo III el más abundante.

La sustitución por radicales acéticos y propiónicos constituye las uroporfirinas, mientras que el reemplazo por radicales metílicos y propiónicos da origen a las coproporfirinas. La protoporfirina es la porfina con sus hidrógenos sustituídos por cuatro radicales metílicos, dos propiónicos y dos vinílicos; constituye el núcleo prostético del Hem, que al unirse con la globina forma la inolécula de hemoglobina.

Las diferentes porfirinas se identifican mediante el estudio cie su espectro de absorción, que es característico para cada una de ellas, habiendo una banda común a todas conocida con el nombre de "banda de Soret”, que identifica el núcleo porfina y ctras adicionales, que dependen de los diferentes radicales de sustitución.

Al disolver porfirinas en ácidos minerales fuertes o en disolventes orgánicos y exponerlos a la luz ultravioleta emiten fluorescencia roja fuerte, característica, propiedad que se utiliza para 
investigar pequeñas cantidades de porfirinas libres. Las coproporfirinas I y III son solubles en mezclas de ácido acético glacial y éter, de los cuales se extraen con ácido clorhídrico. Por otra parte, las uroporfirinas son insolubles en la mezcla ácido-éter, pero parcialmente solubles en acetato de etilo, del cual se extraen igualmente con ácido clorhídrico; estas soluciones clorhídricas dan la fluorescencia roja característica con la luz ultravioleta que sirve para identificarlas.

Biosintesis de las porfirinas.-A partir de dos compuestos básicos, la glicina o glicocola y el "succinato activo", derivado coenzima - A del ácido succínico, se forma el ácido alfa-aminobeta-cetoadípico, el cual por descarboxilación produce el ácido celta-amino-levulínico. Dos moléculas de éste se condensan para formar el porfobilinógeno, a partir del cual se forman en etapas sucesivas las uro-copro y protoporfirinas. Incubando fragmentos ce hígado de pato con ácido delta-amino-levulínico, se han obterido tanto el porfobilinógeno como porfirinas, aunque no se conocen las etapas exactas de las sintesis; algunos autores sostienen que el porofbilinógeno se transforma inicialmente en un compuesto dipirrólico que al condensarse nuevamente da origen a la porfirina. Normalmente se eliminan de 60 a 280 gammas por día cle coproporfirina".--(Tomado del artículo Las Porfirias, del doctor Oscar Gutiérrez R., publicado en la revista El Médico, número 8 , mayo de 1959).

Clasificación.-Teniendo en cuenta el nivel en el cual se prociuce la alteración metabólica y el órgano en que asienta la falla enzimática, Watson divide las porfirias en dos grandes grupos: I. Porfiria eritropoyética y II. Porfiria hepática, la cual, a su vez, se subdivide en: 1. Intermitente aguda; 2. Cutánea tardía; 3. Mixta y 4. Latente, Todas estas variedades tienen como caracteristica común la eliminación de uroporfirinas por la orina y las heces, así como el aumento en la eliminación de coproporfirinas. Quedan fuera de esta clasificación algunas entidades en las cuales hay aumento en la eliminación de coproporfirinas, sin que se encuentren en la orina uroporfirinas ni sus precursores; tales serían la intoxicación por el plomo, la anemia perniciosa, las anemias hemolíticas, la cirrosis portal, la hepatitis, la enfermedad ċe Hodgkin, la neumonia, el absceso pulmonar, la fiebre reumática, etc., en las cuales se producen porfirinurias llamadas sintomáticas. 


\section{SINTOMATOLOGIA}

10 Porfiria eritropoyética.-Esta forma de la enfermedad es congénita, se hereda con carácter recesivo y parece predominar en el sexo masculino. Suele manifestarse desde el nacimiento bajo la forma de eliminación de orina roja oscura. a lo cual se sucede rápidamente la aparición de otros síntomas', tales como fotosensibilidad extrema con producción de eritemas, ampollas, úlceras y hasta mutilaciones ocasionadas por la luz ultravioleta. A causa de la afinidad de la porfirina por el fosfato de calcio, aparece la eritrodoncia y la coloración rosada de los huesos. Los dientes pueden mostrar fluorescencia roja a la luz ultravioleta en los casos en que la eritrodoncia no es muy marcada. Se ha observado también hipertricosis e hiperpigmentación de las zonas expuestas a la luz y, en algunos casos, hepato y esplenomegalia acompañadas de anemia hemolítica. Es de anotarse que en la orina nunca se ha encontrado porfobilinógeno, aunque sí un aumento en las cantidades de uroporfirina y coproporfirina. Basados en la acción fotodinámica intensa de las porfirinas, algunos autores han reproducido experimentalmente los sintomas cutáneos por medio de inyección de porfirinas y exposición a la luz.

20 Porfiria intermitente aguda.-De acuerdo con Waldeström al parecer el autor que más ha estudiado este tema, la porfiria intermitente aguda es la forma clínica más frecuente. Suele presentarse bajo la forma de crisis de dolor abdominal agudo. localizado o generalizado a todo el abdomen, acompañada de vómito, todo lo cual hace que estos pacientes sean frecuente y erróneamente sometidos a tratamiento quirúrgico, a laparotomías de precisión. Al examen clínico es común encontrar gran distensión intestinal, sin contractura ni defensa de la pared hipertermia moderada y leucocitosis con desviación a la izquiercia del leucograma. En ocasiones se encuentra taquicardia e hipertensión arterial, dándosele en la actualidad gran valor a la frecuencia del pulso, en eq sentido de que el regreso a las cifras normales indica mejoría en el cuadro tóxico. El vómito puede concucir a desequilibrios electrolíticos severos. La constipación intestinal es un signo casi constante. Los trastornos neurológicos son notorios, especialmente las neuritis periféricas, parálisis flácidas, paraplejias y parálisis laríngea. Un signo muy sugestivo de porfiria intermitente aguda es la areflexia patelar con persistencia del reflejo aquiliano y clonus del pie. Se han descrito crisis єpileptiformes, aisladas o asociadas al cuadro doloroso abdominal. 
Ios trastornos neurológicos agravan considerablemente el pronóstico, no siendo raros los casos en que la muerte sobrevenga rápiclamente.

"Las orinas son de color rojizo o anaranjado y manchan las ropas de color rojo; esta coloración se debe a la uroporfirina. En ccasiones son de color normal en el momento de la emisión, pero al exponerlas a la luz se oscurecen rápidamente, adquiriendo color ce vino Oporto o de coca-cola, fenómeno debido a la transformación del porfobilinógeno, incoloro, en porfobilina, que es' de color pardo oscuro. El examen clínico muestra grandes cantidades de uroporfirina, así como también porfobilinógeno y ácido deltaamino-levulínico. La presencia de porfobilinógeno es patogonómica de esta afección".

$3^{\circ}$ Porfîria cutánea tardia.-Opina Waldeström que esta forma cie la enfermedad tiene tendencia hereditaria, transmitiéndose con un carácter mendeliano dominante y mostrando clara predilección por el sexo masculino. Los enfermos presentan tardíamente, alrededor de los treinta o cuarenta años de edad, dermopatías consistentes principalmente en ampollas y erosiones en las partes expuestas a la luz; el calor y los traumatismos pueden también producir estas lesiones. Esta forma de la enfermedad se asemeja notablemente a la forma eritropoyética, de la cual se distingue, sin embargo, porque no presenta las alteraciones hemolíticas de la forma eritropoyética y las concentraciones de porfirinas en la medula ósea son normales. En la orina hay uroporfirina pero no porfobilinógeno.

Waldeström distingue dos variedades de la forma cutánea tardía: la hereditaria y la adquirida, presentándose esta última especialmente en casos de lesión hepática severa, bien de tipo cirrótico o tumoral.

4\% Porfiria mixta.-En esta forma es frecuente encontrar sintomas cutáneos, abdominales o nerviosos en forma aisilada o combinada. En la orina se suelen encontrar tanto uroporfirina como corfobilinógeno; la aparición de éste último, coincide generalmente con síntomas abdominales o nerviosos.

$5^{\circ}$ Porfiria latente.-En este grupo están comprendidos los casos en los cuales hay eliminación urinaria de uroporfirinas, sin síntomas clínicos de la enfermedad. "Su identificación tiene importancia profiláctica, a fin de evitar el uso de barbitúricos, sulfonal, alcohol, sulfamidas y otras drogas que podrían desencadenar un ataque agudo". 


\section{ANATOMIA PATOLOGICA}

Generalmente los hallazgos de autopsia son pocos y variacos, así que no puede describirse una entidad patológica, específica asociada a porfiria aguda. Los cambios en el sistema nervioso central, cuando están presentes, son de naturaleza degenerativa, siendo el más notable una desmielinización "a parches". El hígado puede ser de color muy oscuro, agrandado y, microscopicamente, las células hepáticas pueden mostrar alteraciones que varían de la infiltración turbia a la necrosis central. El que los cambios estructurales encontrados, sean consecutivos a los ataques agudos o directamente relacionados y correspondientes a las alteraciones metabólicas básicas, hasta el momento no se ha aclarado, ya que no hay suficiente familiaridad con la estructura histológica del hígado, en las fases latentes de la enfermedad.

En 1954 Schmidt y colaboradores estudiaron los cortes de once biopsias hepáticas y tres hígados post-mortem y encontraron que los cuadros histológicos eran normales en todos, con excepción de uno que perteneció a un paciente en fase latente. El bazo puede estar agrandado con proliferación de células reticulares; los túbulos renales, pueden mostrar infiltración turbia difusa con áreas focales de atrofia. Esto puede también ser notado en los glomérulos. La medula ósea puede aparecer hiperplásica. El hígado, kazo, riñones y medula ósea contienen pigmento marrón, que puede tener o no hierro. Puede notarse marcada atrofia de músculos estriados. Las estriaciones cruzadas del músculo cardíaco se tiñen pobremente y puede haber allí una infiltración de células redondas, agregadas a la degeneración grasosa de las fibras musculares mismas.

\section{DIAGNOSTICO}

En las formas eritropoyéticas y cutánea tardía de la porfiria generalmente no hay mayor dificultad diagnóstica, ya que presentan manifestaciones bastante características que orientan al clínico hacia el diagnóstico exacto, el cual se corrobora al investigar la presencia de uroporfirina en orina. En la forma intermitente aguda, por el contrario, el polimorfismo de sus manifestaciones hace que a menudo sea confundida con enticlades de patología quirúrgica abdominal de urgencia, llegándose solamente al diagnóstico correcto después de una o de varias' laparotomías en blanco. Debe, sin embargo, pensarse en porfiria intermitente aguda siempre que se encuentre un cuadro abdominal 
agudo, con náuseas, vómitos y constipación pero sin contractura rnuscular de la pared abdominal y ausencia de sensibilidad a la palpación y compresión. El diagnóstico en estos casos lo hace el hallazgo de porfobilinógeno en orina, para cuya investigación se aconseja la prueba de Watson-Schwartz, que consiste en lo siguiente: a 1 c. c. de orina fresca agréguese 1 c. c. de reactivo de Ehrlich. Mezclar. Agregar 2 c. c. de solución supersaturada químicamente pura de acetato de sodio. Mezclar. Agregar 5 c. c. de cloroformo; mezclar fuertemente; dejar que la mezcla se separe en fase acuosa y cloroformo; el porfobilinógeno, si está presente, formará un aldehido marrón-rojo al añadirle reactivo de Ehrlich, el cual permanecerá en la capa acuosa después de mezclarlo con el cloroformo.

En las formas mixtas de porfiria, las lesiones cutáneas características acompañadas de hipertricosis e hiperpigmentación, se distinguen por la eliminación de uroporfirinas, en tanto que en los cuadros abdominales y en los de predominio neurológico suele encontrarse porfobilinógeno.

La forma latente de la porfiria se diagnostica por la presencia de uroporfirina y aun de porfobilinógeno en la orina.

Pronóstico.-El pronóstico depende de la forma clínica de porfiria que se contemple. En la forma eritropoyética y cutánea tardía, si bien pueden dejar lesiones permanentes como las mutilaciones, no comprometen seriamente, en cambio, la vida del paciente. En la forma intermitente aguda, por el contrario, alguros autores dan cifras de mortalidad hasta del $20 \%$ en los cuadros abdominales y hasta del $90 \%$ en los casos con compromiso nervioso. En la porfiria mixta, como se comprende, el pronóstico depende de la naturaleza de los síntomas que predominen en el cuadro clínico; si hay predominio de síntomas cutáneos, el proróstico es bueno; no lo es, en cambio, en las formas mixtas en que hay predominio de síntomas abdominales o nerviosos.

\section{TRATAMIENTO}

Porfiria eritropoyéticas.-En esta forma de la enfermedad el tratamiento es principalmente profiláctico, evitando el exposición del enfermo a los rayos solares; Waldenström ha sugerido la conveniencia de trabajar de noche y evitar la luz durante el día. Algnos casos complicados con anemia hemolítica han sido tratados con esplenectomía; los resultados obtenidos, sin embargo, no permiten sacar ninguna conclusión todavía. 
Porfiria intermitente aguda: Infortunadamente en esta forma clínica de la porfiria, la más grave de todas, no existe ningún acuerdo entre los diversos autores en lo que a su tratamiento se refiere. Es así como Marañón indica el ácido nicotínico, Von Domarus el complejo B a altas dosis y el ACTH, Peters y colaboradores el Bal y el Edta (etilene-diamina-tetraaceato calcio sódico), otros los derivados de la fenotiazina, Tricomi y Baum el Diemtrol, vigilando el balance hídrico y electrolítico durante el ataque agudo, etc. Todos, sin embargo, están de acuerdo en que se debe hacer un tratamiento sintomático, preconizando algunos el empleo de enema's y purgantes para combatir la constipación. liegándose inclusive a la utilización de la prostigmina. Se ha empleado también, la esplacnicectomía bilateral en vista del buen resultado que en ocasiones se obtiene con el bloqueo ganglionar. I os resultados obtenidos con toda esta serie de tratamientos han sido, sin embargo, inconstantes.

Porfiria cutánea tardía: Se aconsejan medidas de protección contra el sol, vigilar el buen funcionamiento hepático y alimentación balanceada. Respecto del empleo de medicamentos en esta forma de la porfiria no existe unidad de criterio alguna.

Porfiria latente: Evitar el uso de tóxicos, tales como los barbitúricos, el alcohol, el sulfonal, las sulfamidas, etc., que podrían cesencadenar un ataque agudo.

\section{Presentación de un caso}

Nombre: Y. V. de R. Edad: treinta y un años. Natural de Fopayán. Fecha de inscripción: 30-IX-58. Estado civil: casada. Residencia: Bogotá. Edad matrimonial: 4 años.

Motivo de consulta.-La paciente llega al consultorio particular para control prenatal. Grávida IV. Para Ir. Aborto I.

Antecedentes familiares: Sin importancia para el caso.

Antecedentes personales: Sarampión, tifoidea y parotiditis, en la infancia.

Antecedentes quirúrgicos: Negativos.

Antecedentes circulatorios: Dolores precordiales ocasionales. Antecedentes respiratorios: Negativos.

Antecedentes digestivos: Constipación crónica que, según ceclara la paciente, mejora con los embarazos. Antecedentes de cos cólicos intestinales muy fuertes' en años anteriores, sin poder precisar fechas. 
Antecedentes urinarios: Negativos.

Antecedentes nerviosos: Negativos.

Organos de los sentidos: Anginas frecuentes.

Antecedentes ginecológicos: Menarquía a los catorce años. Ciclos normales de 28/IV a VI. Dismenorrea (congestiva?).

Antecedentes obstétricos: Tres embarazos anteriores El pririero de término; dos inducciones; trabajo de 72 horas; distocia cervical; parto espontáneo; feto femenino de 3.100 gramos; puerperio normal. Segundo: Aborto completo de dos meses. Tercero: De término; hizo un falso trabajo; trabajo de tres horas, refor. zado con Pitocín; parto espontáneo; feto masculino, vivo, de 3.300 gramos; puerperio normal; fecha del último parto: 23-II-57.

Embarazo actual: Fecha de la última menstruación: 16-III-58. Fecha probable del parto: 24-XII-58. El embarazo ha evolucionado sin complicaciones, salvo uria discreta sensación de astenia y edema de miembros inferiores ocasionales, principalmente vespertinos.

Examen fisico.-Examen general: Talla: 1,67 metros. Peso: 75,5 kilos. Inspección general: Discreta hipertrofia tiroidea.

Aparato circulatorio: Corazón clínicamente normal. Tensión arterial: 130/90. Pulso: 88 al minuto.

Aparato respiratorio: Clínicamente normal.

Aparato digestivo: Lengua limpia. Prótesis dental. Abdomen regativo a la exploración del aparato digestivo.

Aparato urinario: Negativo a la exploración.

Sistema nervioso: Moderada hiporreflexia tendinosa.

Aparato osteo-muscular: Normal.

Organos de los' sentidos: Normales.

Examen obstétrico.-Altura uterina: 21 centímetros. Anomalias del útero: No. Número de fetos: Unico. Desarollo: 6 meses. Presentación: Cefálica. Auscultación fetal: positiva en fosa ilíaca derecha. Pelvimetría: Biespinoso: 25 centímetros. Bicrestílico: 31 centímetros. Bitrocantéreo: 33 centimetros. Baudelocq: 21 centímetros. T. vaginal: Genitales externos normales. Cuello corto, blanco, cerrado. Fondos de saco vaginales libres.

Se prescriben laxantes del tipo del aceite mineral para combatir el estreñimiento y se solicitan exámenes de laboratorio de nutina.

27-X-58. Consulta a las 11:00 por cólico intestinal muy fuerte que comenzó a las 22:00 del día anterior. Contracciones uterinas 
irregulares. El esposo, que es médico, le ha dado 30 gotas de láuclano y le ha aplicado 2 ampollas de Atrinal vía intramuscular, sin resultado. Al examen solo se aprecia un meteorismo moderado; el cuello uterino no ha sufrido modificación alguna. T. A : 125/80. Pulso: 100 . Temperatura oral: $36^{\circ} 8$; rectal: $37^{\circ}$. Se decide insistir en los antiespasmódicos y tener a la paciente en observación. A las 17:00 consulta nuevamente. El dolor abdominal se ha intensificado notablemente. El cuello uterino continúa sin modificación. Hay gran distensión intestinal. Ha vomitado dos veces en las horas de la tarde: el vómito es de aspecto bilioso. Se convoca junta médica con el gastroenterólogo, quien hace diagnóstico de impresión de oclusión intestinal; se decide la hospitalización de la paciente en la Clínica de Marly. Allí se procede a tomar placa simple de abdomen, que resulta negativa para oclusión intestinal. Un cuadro hemático hecho de urgencia revela una leucocitosis de 20.300 con neutrofilia de 81 y $3 \%$ de cayados. Ante la posibilidad de un absceso apendicular que esté determinando el cuadro abdominal agudo se decide practicar laparotomía de precisión, que resulta completamente negativa después de prolija exploración de la cavidad abdominal; solo se encuentra una enorme distensión del ileon y el cólon. Se cierra la pared en planos, se colocan puntos ce tensión y se prescribe succión intestinal continua, cloramfenicol, betaglicerofosfato de sodio como prevención del parto prematuro, morfina e hidratación con solución electrolítica Travert número 1. La intervención se practicó el 28-X-58 a las 2:00, con duración de 1 hora; anestesia general con ciclopropano-oxígeno.

29-X-58. Continúa la distensión abdominal. Paciente afebril. Se continúa la prescripción del día anterior, con Travert $10 \%$ solución electrolítica número 2 y Edhanol 1 ampolla cada 6 horas. Fn las horas de la tarde, además de lo ordenado, se le indica Prostigmine 1 ampolla cada 6 horas y Demerol 100 miligramos cada 6 horas, alternando con anterior. Ante este cuadro se sugiere la posibilidad de una porfiria.

30-X-58. Comienza a ceder la sintomatología. Se continúan las mismas prescripciones. Se han ordenado además, lavados intestinales (con poco resultado) y aceite mineral por la sonda gástrica. El día anterior le fue practicado un examen de orina que reveló: albúmina 0.45 gramos por ciento; cilindros hialinos ${ }^{++}$; hialinosgranulosos ++ y granulosos ++ ; glóbulos rojos ++ ; pigmentos biliares $+=$. Se toma orina de 12 horas obtenida por cateterismo (a permanencia), 300 c. c. para investigación de porfirinas. El 
dolor ha disminuído apreciablemente. Auscultación fetal +. Movimientos fetales + .

31-X-58. La distensión abdominal ha disminuído en un $60 \%$. Continúa afebril. El examen para porfirinas da el siguiente resultado: Uroporfirina ++ ; Coproporfirina + ; Porfobilinógeno + (Técnica de Waldenström). Los controles de azohemia son normales; cuadro hemático normal con 7.000 leucocitos; neutrófilos 69\%; linfocitos $27 \%$. La sedimentación está en $45 \mathrm{~mm}$. a la hora. En la crina la albúmina ha descendido a huellas y los cilindros a granulosos +; persisten los glóbulos rojos ++ . Dice haber tenido contracciones uterinas, pero al T. V. el cuello no se ha modificado. Con diagnóstico de Porfiria aguda (en fase ya de regresión) se ordena: ACTH 40 miligramos diarios IM; ácido fólico, 10 miligramos diarios IM. Travert 10\%; solución electrolítica número 1 , 1.000 c. c. IV: Suspendiendo la anterior medicación.

1-XI-58. Se continúan las mismas prescripciones del día anterior; al igual que Prostigmine, que venía aplicándose regularmente. Se retira sonda gástrica. Abdomen normal. Se ordena levantarse.

3-XI-58. Paciente en buenas condiciones. Se retiraron puntos de piel, dejando únicamente los de tensión. Hay muy ligero meteorismo. Funcionamiento intestinal normal, previa aplicación de lavado con aceitie de ricino; expulsión de gases satisiactoria.

Paciente afebril. Anoréxica (desde el día anterior se ordenó alimentación completa). Continúa con ACTH. Folbessin y ácido nicotínico. Feto en O. I. alto. Auscultación fetal +. Dice que la víspera, al levantarse, hizo lipotimia pasajera.

4-XI-58. Sale de la Clínica en buenas condiciones. No se han retirado los puntos de tensión. Se suspende el ACTH y se continúa solo el ácido nicotínico.

7-XI-58. Visita a domicilio con el gastroenterólogo. Se queja de dolor en la herida quirúrgica; los puntos de tensión producen fuerte dolor. Muy buen estado general. El intestino ha funcionado espontánea y satisfactoriamente; el apetito ha mejorado notablemente. T. A.: 120/80. Afebril. Feto en O. I. alto. RF +. Se decide retirar los puntos de tensión el día 10-XI-58. Continúa tomando Ei ácido nicotínico; se queja de cefaleas ocasionales. El día anterior le fue practicada una nueva investigación de porfirinas en crina, con resultado negativo. El informe dice que la orina aún Está coloreada, pero no tanto como en días anteriores. 
10-XI-58. Se retiran los puntos de tensión; pared abdominal en buenas condiciones; sutura cicatrizada. Se suspende el ácido nicotínico. Paciente en muy buenas condiciones.

22-XI-58. Control de orina: Porfirinas: negativo. Densidad: 1.017. Albúmina: ligeras huellas.

27-XI-58. Orina: Porfirinas: negativo. Albúmina: ligerísimas huellas.

27-XI-58. Altura uterina: 27 centímetros. T. A.: 115/80. Edemas inferiores ${ }^{++}$. Cicatrización completa de la laparotomía. $\mathrm{RF}+$. Paciente en magníficas condiciones.

1-XII-58. Proteinemia: 5 gramos por ciento; albúminas 3,20 gramos; globulinas: 1,80 gramos por ciento. Persisten los edemas Se ordena dieta hiposódica; Ledinac 3 cucharadas al día. Orina: Densidad 1.017. Albúmina: muy ligeras huellas. Porfirinas (-). Sedimento: leucocitos ${ }^{+}$; piocitos ${ }^{+}$; células epiteliales ${ }^{++}$; cristales + ; bacteria +++ .

5-XII-58. (Visita a domicilio). Los edemas han desaparecido casi totalmente. Estado general satisfactorio. T. A.: 120/80. RF +. Discreto dolor sobre la cicatriz quirúrgica. Constipación intestinal pertinaz; tiene que tomar laxante con aceite mineral para poder cefecar. No se ha podido controlar el peso de la paciente. Se crdena continuar dieta hipasódica y Ledinac.

16-XII-58. Control en consultorio. Examen de orina del 10XII-58. Densidad: 1.015. Ph: 6. Hematíes: no hay. Leucocitos: $+_{+++}$; células epiteliales ${ }^{++}$; cilindros no hay; cristales: uratos amorfos + ; bacterias + . Albúmina y glucosa (-). Pigmentos biliares: escasos.

Se queja de disuria desde hace unos 15 días. Dice que el color de la orina continúa oscuro. Digestión ayudada con aceite mineral; constipación moderada. Dolores abdominales difusos frecuentes; cefalea occipital; dice que su visión es normal, con excepción de fosfenos ocasionales. Apetito aceptable, a pesar de estar comiendo solo verduras. Los pies se han deshinchado apreciablemente, pero no del todo. Acedías fuertes; náuseas ocasionales. Dolor sacro y sinfisalgia. Dolor discreto sobre la herida quirúrgica.

Examen.-T. A.: 135/90. Pulso: 84. Peso: 76 kilos. A. U.: 31 centímetros. O. D. fijo RF +. Se ordena: suspender Ledinac. Dayamineral, 3 comprimidos al día. Control de T. A. cada 2 días. Disuria marcada. Pyridium, 3 comprimidos diarios. 
19-XII-58. Comienza trabajo de parto irregular a las 4:00; a las 11:30. T. V.: cuello delgado, duro; discreto edema de labio anterior. Dilatación 5 centrímetros. O. D. alto. $\mathrm{RF}+$. Contracciones cada 7 minutos, de mala intensidad. Se ordena goteo con $3 \mathrm{U}$. de Fitocín en 500 c. c. de Dextrosa al 5\% en agua destilada, a 15 gotas por minuto. Largactil media ampolla IM.

12:45: Demerol, 50 miligramos. IM.

14:00 T. V.: cuello borrado, blando, dilatado 8 centímetros. Bolsa integra. O. D. alto. $\mathrm{RF}+$. Excitación. Continúa el goteo; contracciones cada 4 minutos, de regular intensidad. Se pasa a la sala. Analgesia "a la reina" con ciclo-oxígeno.

14:45. Ruptura artificial de las membranas; líquido amniótico ligeramente teñido de meconio; se da oxígeno y se agregan 5 U. de Pitocín a la dextrosa.

15:05. Dilatación completa. O. D. T. en estrecho medio. La paciente no colabora. Se da anestesia con ciclopropano-oxígeno Y se hace aplicación de fórceps de simpson para O. D. T. en estrecho inferior.

15:10. Parto de una niña de 3.200 gramos de peso y 48 centimetros de talla. Sin lesión congénita aparente. Respiración espontánea.

15:15. Extracción manual de la placenta y revisión manual del itero que se encuentra normal. Se ha producido un desgarro perineal del $2^{\circ}$ grado que se sutura en planos con catgut simple número 1. Peso de la placenta 700 gramos. Toques de merthiolate en sutura perineal dos veces al día. Methergin 5 gotos dos veces a! día.

20-XII-58. Mismas indicaciones. Puerperio normal.

21-XII-58. Se suspende Methergin. Lavado intestinal.

22-XII-58. Sutura perineal cicatrizada. Sale de la Clínica en buenas condiciones.

El parto se realizó en la Clínica de Marly. La paciente no concurrió a control post-natal y, desgraciadamente, por falta de su colaboración, no fue posible hacerle investigación de porfirinas en orina después del parto, tanto a ella como a la niña.

\section{Comentario}

A causa de la naturaleza de la porfiria intermitente aguda; una enfermedad caracterizada por períodos de exacerbaciones y remisiones espontáneas, estamos de acuerdo con O'Dwyer en que es difícil evaluar el efecto del embarazo sobre su curso natural. 
Sin embargo, de los casos detallados reportados en la literatura, podemos concluir que si bien ei embarazo no tiene un definido efecto benéfico sobre la enfermedad, tampoco la agrava. Como corolario, la enfermedad no parece tener un efecto adverso o verjudicial sobre el embarazo, excepción hecha, claro está, de las complicaciones a que pueda dar lugar una intervención quirúrgica realizada por error de diagnóstico, como sucedió en el caso que nos permitimos presentar.

\section{BIBLIOGRAFIA}

GUTIERREZ, OSCAR.- "Las Porfirias. Revisión del problema y presentación de dos casos". "El Mécỉico", número 8; 1959; páginas 8-15.

TRICOMI, VICENT; EAUM, HAROLD.- "Acute Intermitent Porphyria and Fregnancy'. Obstetrical and Ginecological Survey, número 3, volumen XIII, página 307. 1958.

WATSON, C. J.; SCHWARTZ, S.-“A simple test for urinary porphobilinogen”. Proc. Soc. Exper. Biol. \& Med., 47: 393, 1941.

FREEDMAN, A., YEAGLY, J. D. and BROOKS, J. B.-."Acute Porphyria with Improvement during and following Pregnancy". Am. Int. Med., 36: 1.111, 1952.

DURST, J. B. and KREMBS, H. A.--"Porphyria and Pregnancy". J. A. M. A, 160: 3, 1956.

O'DWYER, J. P.-“Acute Iidiopathic Porphyria Complicating Pergnancy”. J. Obst. \& Gynec. Brit. Emp., 63: 437, 1955.

MARAÑON, G:-Manual de Diagnóstico Etiológico. $5^{\text {a }}$ edición, EspasaCalpe, 1950. Páginas 556-558.

HOLLER, G. y PFLEGER, R.-Sintomatología de las enfermedades internas y su valoración para el diagnóstico diferencial. Jabor, Reimpresion. 1946. Páginas 748 y 749 . 